\title{
Co-Creation bei komplexen Consumer Products
}

Linda Geißler, Nico Herzberg, Natalie Mundt

Endkunden legen bei ihren Kaufentscheidungen immer mehr Wert auf Individualität. Als Reaktion darauf, haben Unternehmen das Potential der Co-Creation erkannt. Dies beschreibt die Zusammenstellung eines komplexen Produktes durch den Kunden selbst. Auch Campeleon nutzt Co-Creation im Rahmen der Produktentwicklung. Das Start Up entwickelt in enger Zusammenarbeit mit Pilotkunden modulare Ausstattungsmodule für Reisemobile. Durch die intensive Interaktion mit dem Kunden konnten Anforderungen an die Produkte identifiziert werden, die ansonsten unentdeckt geblieben wären. So sind erste modulare Ausstattungsmodule entstanden, die es ermöglichen, den knappen Platz im Innenraum von Reisemobilen durch Mehrfachnutzung der Flächen zu maximieren. Neben einer innovativen Küchenlösung ist so auch ein modulares Camper-Bad entstanden. Gleichzeitig muss sich Campeleon immer wieder die Frage stellen, in welchem Maße Co-Creation sinnvoll genutzt werden kann und wann sie für den Kunden überfordernd wirkt. Daraus ist eine Checkliste entstanden, die Kunden dabei behilflich sein soll, ihre Gedanken zum Reisemobil-Ausbau zu ordnen. Dieser Artikel beleuchtet zum einen Vorteile und Herausforderungen von Co-Creation. Zum anderen wird die Entstehung eines Prozesses zur Umsetzung anhand eines Anwendungsbeispiels veranschaulicht.

Keywords: Co-Creation. iterative Produktentwicklung

\section{Vor- und Nachteile von Co-Creation}

Neue technologische Entwicklungen und die sich stetig ändernden Erwartungen von Endkunden (Consumer) eröffnen neue und vielfältige Möglichkeiten, Produkte zu kreieren und neu zu interpretieren. Der Endkunde legt beim Produktkauf zunehmend Wert auf Individualität und Selbstbestimmung. Gleichzeitig ist er bereit, sich im Entstehungsprozess einzubringen. Die sogenannte Co-Creation hat einen positiven Effekt auf das Unternehmensbild und stärkt die emotionale Bindung zum Produkt (Bendapudi, N. \& Robert P. L.:2003). Zusätzlich erhöht die Einbindung zukünftiger Kunden in die Produktentwicklung die Chancen auf ein am Markt erfolgreiches Produkt.

Im Gegensatz dazu kann das Einbeziehen der Kunden in die Produktzusammenstellung insbesondere bei komplexen Produkten allerdings auch zu Überforderung führen (Scheibehenne, B., Greifeneder R. \& Todd P. M.:2010). Daher ist es wichtig, den richten 
Grad der Einbeziehung des Endkunden in die Produktentwicklung und die Zusammenstellung des Produktes zu finden oder diese durch den Kunden wählbar zu gestalten.

In diesem Beitrag unterscheiden wir hinsichtlich:

(a) Co-Creation bei der Entwicklung der eigentlichen Produkte, die später ihren Weg in das Angebot finden. Bspw. können Endkunden in die Entwicklung bestimmter Möbellösungen in einem Reisemobil einbezogen werden und aktiv Ideen und Vorschläge bis hin zu kompletten Produktentwürfen einbringen.

(b) Co-Creation bei der Zusammenstellung eines komplexen Produkts durch den Endkunden selbst, welches er am Ende käuflich erwirbt. Bspw. kann ein Kunde sein Reisemobil aus verschiedenen Bausteinen zusammenstellen und so ein individuelles und auf seine Bedürfnisse abgestimmtes Produkt erwerben.

Im weiteren Verlauf des Beitrags konzentrieren wir uns auf den letzteren Anwendungsfall in der Produktzusammenstellung (b) und geben nur im Ausblick, (siehe Kapitel 5) eine kurze Indikation zu Co-Creation in der Entwicklung von Produkten (a) selbst.

\section{Anwendungsfall}

Campeleon - The Art of Camping ist ein junges Dresdner Unternehmen, das die digitale Produktentwicklung als Schlüssel zum Erfolg nutzt. Im Zentrum steht ein digitaler 3D-Konfigurator mit dessen Hilfe Endkunden auf Basis modularer Ausbaumöbel ihren individuellen Campervan zusammenstellen können.

Campeleon versteht sich als IT-Unternehmen mit einem physischen Produkt. Alle Geschäftsprozesse laufen über eine digitale Plattform ab - der Kundenkontakt, der Kaufprozess, die unternehmensinterne Datenverarbeitung und die Generierung und Weitergabe der Produktionsdaten an das Produzenten- und Montagenetzwerk.

Fundament dafür ist ein von Campeleon entwickelte modulare Ausbausystem für Campervans. Es basiert auf einem in das Basisfahrzeug eingepasste Anschlusssystem und flexibel anbringbaren Ausstattungsmodulen. Das Anschlusssystem ist so konzipiert, dass es in verschieden Fahrzeughöhen und -längen eingepasst werden kann und auch für verschiedene Fahrzeugkonturen funktioniert. Durch eine Normierung der Innenkontur und einem festen Rastermaß, können die Ausstattungsmodule unabhängig von der Fahrzeugbasis entwickelt werden. Der Innenausbau selbst besteht aus einer Vielzahl von Einzelprodukten - den Ausstattungsmodulen wie Küchen-, Bad-, Stauraum-, Sitz- und Schlafmodulen sowie Abdeckmodulen. Sie sind in ihren Dimensionen, Funktionen und der Konstruktion standardisiert. So können sie variabel kombiniert, positioniert und getauscht werden. Die Ausbaumodule können außerdem in andere 
Fahrzeuge mit dem passenden Anschlusssystem mitgenommen werden, sodass ein defektes Fahrzeug nicht gleich das Ende des gesamten Campervans bedeutet. Der Ausbau zieht einfach mit um.

Campeleon verfolgt den Weg eines nachhaltigen und transparenten Unternehmens mit einer offenen Kommunikation. Dabei ist die frühzeitige Einbindung von Interessierten und möglichen Kunden in die Produktentwicklung ein Mehrwert auf verschiedenen Ebenen - Marketing und Vertrieb, User Research und Open Innovation, Produktion und Partnernetzwerk. Wenn es darum geht, Produkte mit einem nachhaltigen Wert für die Kunden zu schaffen, ist deren persönliches Investment erforderlich. Mit der Höhe des individuellen Aufwandes in eine Aktivität steigt zugleich die emotionale Bindung [1]. Dabei stellen sich folgende Fragen, die diskutiert werden müssen:

- Über welche Kanäle (digital, aber auch analog) werden die Endkunden involviert?

- In welcher Intensität werden die Kunden in die Produktentwicklung und die Zusammenstellung ihres eigenen individuellen Produktes involviert?

- In welcher Komplexität können die Kunden involvieren werden?

- Muss man die Kundenzielgruppe hinsichtlich Intensität und Komplexität der Involvierung unterschieden?

- Wie kann der Prozess der Co-Creation strukturiert werden?

\section{Co-Creation bei der Zusammenstellung des Endprodukts}

Einer der globalen Megatrends ist die Individualisierung (Zukunftsinstitut 2020). Auch im Konsum und in den konsumierten Produkten soll sich der Individualismus des Einzelnen wiederfinden. Eine Möglichkeit, dies umzusetzen ist es, nach dem DIY (Do It Yourself) Trend selbst Produkte zu erschaffen oder individualisierte Produkte zu kaufen. Als Beispiel kann hier das Personalisieren der eigenen Schuhe, wie es u.a. bei Nike und Adidas möglich ist, anzuführen.

Im Bereich der Reisemobile hat sich bisher nur DIY als Möglichkeit der Individualisierung ergeben. Viele Menschen, die das Reisen im Campervan für sich entdeckt haben, bauen einen Kastenwagen nach ihren Wünschen aus oder beauftragen eine Manufaktur. Die breite Masse kann damit nicht erreicht werden. Den meisten fehlt Zeit, Platz oder handwerkliches Geschick, um ein komplexes Produkt wie ein Reisemobil individuell und flexibel umzusetzen. 
Bei den klassischen Reisemobilen gibt es für die Kunden eine riesige Auswahl an Fahrzeugen und Grundrissen. Schaut man genauer hin, sind diese so optimiert, dass sie für eine möglichst große Gruppe von Kundenanforderungen passt. Dabei begrenzt sich die Individualisierbarkeit auf Polsterbezüge und Griffformen. Das reicht vielen Kunden heutzutage nicht mehr.

Daher setzt Campeleon darauf, seinen Prozess zur Co-Creation gemeinsam mit den ersten Kunden zu entwickeln. Bei der Entstehung dieses Prozesses wird deutlich, welche Anforderung die Interessenten haben, wo sie überfordert sind und bis zu welchem Grad sie sich einbringen können. Im Folgenden wird beschrieben, wie die Co-Creation in der Interaktion mit Pilotkunden (weiter-) entwickelt wird. Außerdem wird beleuchtet, wie dieser Prozess standardisiert und mittels einer Softwarelösung abgebildet werden kann.

\section{Co-Creation mit Pilotkunden}

Das Ausbausystem basierend auf dem Baukastenprinzip ermöglicht eine unbegrenzt scheinende Anzahl an Möglichkeiten, den Innenausbau zu gestalten und einzelne Ausstattungsmodule zu entwickeln. In der Produktentwicklung stellt sich damit die Herausforderung, entscheiden zu müssen, mit welcher Priorität die jeweiligen Varianten der Module entwickelt werden. Die Basis dafür können nur die Kunden schaffen.

Eine Möglichkeit besteht darin, sich die potenzielle Kundengruppe genau anzuschauen, indem demografische Daten ausgewertet, das Reise- und Konsumverhalten oder Beiträge in den sozialen Netzwerken untersucht werden. Außerdem können Befragungen und Interviews durchgeführt werden. Die erhobenen Daten geben trotz allem nur einen groben Überblick über die Benutzeranforderungen und repräsentieren eine eher inhomogene Kundengruppe.

Campeleon hat sich bei der Auswahl und den Funktionen der ersten Ausstattungsmodule für eine Produktentwicklung mit Pilotkunden entschieden. In Zusammenarbeit mit drei Pilotkundin werden so die unterschiedlichen Anforderungen an einen Campervan in Erfahrung gebracht. Die sehr individuellen und teils stark voneinander abweichenden Wünsche stellen Campeleon vor die notwendigen Herausforderungen, um innovative und kreative Lösungen zu finden. So entstehen unterschiedliche Ausbaugrundrisse, die sich an den individuellen Anforderungen orientieren.

Daher ist es notwendig, Kundenwünsche strukturiert aufzunehmen und die Gedanken der Kunden zu ordnen. Vielfältige Informationen zu Campervan-Ausbauten, die im Internet und besonders über Social Media wie Instagram verfügbar sind, überfrachten die Endkunden mit unstrukturierten Informationen. Diese Erkenntnis konnte nur aus 
der Zusammenarbeit mit den Pilotkunden entstehen. Im nächsten Schritt hat Campeleon einen Prozess entwickelt, mit dem die Kundenanforderungen aufgenommen werden können.

Des Weiteren hat sich aus den Kundeninteraktionen herauskristallisiert, dass es innovative Produkte bedarf, insbesondere in den Bereichen Bad- als auch Küchenlösung. Der Wunsch, Flächen mehrfach je nach situativem Bedürfnis nutzen zu können, ist besonders klar hervorgetreten. Dadurch ist eine Lösung entstanden, die es erlaubt, beim Zubereiten von Speisen mehr Platz als Küche zur Verfügung zu haben. Diese Fläche kann verkleinert werden, wenn sie nicht gebraucht wird - um sie als Bad zu nutzen.

\section{Ergebnisse der bisherigen Co-Creation}

Aus der bisherigen Zusammenarbeit mit den Pilotkunden sind bereits erste Ergebnisse entstanden. Durch die Interaktion mit den Pilotkunden konnten Prozesse optimiert werden. Diese können weiteren potentiellen Kunden digital zur Verfügung gestellt werden. Des Weiteren haben sich neue innovative Produkte ergeben, die nach dem Design und der Konstruktion allen Kunden zur Verfügung gestellt werden.

\section{Checkliste für Kunden}

Campeleon zieht auf eine Zielgruppe ab, die das Reisen im Campervan neu entdeckt und nicht unbedingt auf viele Erfahrungen aus vorangegangenen Campingreisen zurückgreifen kann. Aus der Erkenntnis heraus, dass die Kunden zudem mit einem Kopf bzw. Bildspeicher voller Impressionen aus den sozialen Medien bei einem CampervanAusbau starten, hat Campeleon es geschafft, eine Art Checkliste zu standardisieren. Diese dient als Entscheidungshilfe mit der abgefragt wird, welche tatsächlichen Bedürfnisse hinsichtlich der Ausstattung wie Bad, Küche, Stauraum, Schlafen, Wohnen und auch Transport von Hobby-Equipment bestehen. Das soll den Kunden als Orientierungshilfe dienen, um sie zu ihrem passenden Campervan zu führen. Die Grundlage für die Struktur und die einzelnen Punkte der Checkliste sind in der Kommunikation mit den Pilotkunden entstanden und werden fortlaufend weiterentwickelt. Die Checkliste wird so aufgearbeitet, dass sie online gestellt und von Interessenten und potenziellen Kunden benutzt werden kann. Basierend auf diesen Ergebnissen kann Campeleon ein Grundrisskonzept erstellen und dem Kunden zur Verfügung stellen. Damit gibt es ein erstes Dienstleistungsprodukt, das angeboten werden kann. 


\section{Modulare Küche}

In der Zusammenarbeit mit den Pilotkunden hat sich herauskristallisiert, dass es nicht ausreicht, Module ausschließlich nach ihrer Funktion - wie z.B. Küche, Bad, Stauraum - einzuteilen. Für ein konsequent modularisiertes Konzept müssen auch die Module in sich entsprechend gestaltet werden. Die Anforderungen an die Küche unterscheiden sich in ihrem Umfang (minimale, mittlere und viele Funktionen) und in ihrer Anordnung im Fahrzeug. Im Austausch mit den Kunden wurde aufgezeigt, wie unterschiedlich die Anforderungen an eine Camperküche sein können. Entsprechend hat Campeleon sich dazu entschieden, diese aus einzelnen Modulen aufzubauen, die verschieden kombiniert und an verschiedene Positionen im Fahrzeug platziert werden können. Damit entsteht eine große Varianz an individuellen Küchenlösungen, die verschiedenen Anforderungen entsprechen. Von der Anwendung für einen Hobbykoch mit großem Bedarf an Arbeitsfläche, Stauraum und auch entsprechendem Equipment zum Kochen selbst bis hin zur Mini-Küche für den kleinen Campervan.

\section{Pop-Up Bad}

Gemeinsam mit den Kunden steht Campeleon immer wieder vor der Diskrepanz zwischen einem Fahrzeug mit möglichst geringen Außenmaßen und maximalem Platz im Inneren. Eine Lösung, die das Gefühl von mehr Platz in Inneren vermittelt, ist die mehrfache Nutzung des vorhandenen Raums. Je nachdem, wofür der Raum in diesem Moment genutzt werden soll, lässt sich der Innenraum verändern, um die entsprechenden Funktionen und den Bewegungsbereich zugänglich zu machen. Bei den Schlaflösungen bspw. gibt es diese Ansätze bereits - am Tag eine Sitzgruppe, in der Nacht zum Doppelbett umgewandelt. Doch für das Bad als abgeschlossenen Raum, ist das bisher nicht umgesetzt worden.

Durch die Vorgaben der Pilotkunden hinsichtlich ihrer Bedürfnisse an ein Camperbad, ist das Ausstattungsmodul eines Pop-Up-Bades entstanden. Dies ermöglicht, den Platz für die Ausstattung eines Bades im Campervan auf ein Minimales zu reduzieren, wenn kein Bedarf an einer Badnutzung besteht. Wird das Bad für Hygiene oder einen Toilettengang gebraucht, wird es entsprechend aufgefahren und nutzt Raum, der gerade nicht gebraucht wird - zum Beispiel den Bewegungsraum an der Küche oder den von Passagierfahrsitzen. 


\section{Fazit und Ausblick}

Die Potenziale der Kombination aus physischem Produkt und digitalem Konfigurator sind aus Unternehmenssicht für die Produktentwicklung und die Produktionsabläufe immens. Aus Sicht der Produktentwicklung bietet sich über die digitale Plattform die Möglichkeit, neue Produktideen zu Ausbaumodulen bereits in einer frühen Phase digital zu testen. Das ist kostengünstig und schnell. In kurzen Zyklen kann ein entsprechendes Ausbaumodul modifiziert und wieder getestet werden. Zusätzlich können aus der digitalen Abbildung der Reisemobilausbauten - sog. digitale Zwillinge - neue Erkenntnisse gewonnen werden, die direkt in die Produktentwicklung einfließen können. Da alle Daten in der Plattform vorliegen, kann während des Produktions- und Montageprozesses eine detaillierte Transparenz für den Endkunden geschaffen werden. Dabei bleibt immer die eine spannende Frage: Wieviel Einbezug ist für den Endkunden richtig und damit für die Customer Experience optimal?

Co-Creation kann auch ganz implizit entstehen. Durch die Nutzung des Campeleon 3DKonfigurators entstehen unzählige Datenmengen aus denen Informationen zur Nachfrage nach bestimmten Produkten, zur Anordnung der einzelnen Module usw. gewonnen werden können. Durch intelligente Auswertung dieser Daten kann spezifischer auf den Kundenbedarf eingegangen werden. Auch auf diesem Weg bringen sich die Kunden in die Produktentwicklung ein.

Die einzelnen Campeleon Ausstattungsmodule sind ausschließlich vom standardisierten System abhängig. Die Variablen des Systems stellen die Rastermaße und die Befestigung an sich dar. Dadurch bietet sich für Campeleon eine weitere Form der CoCreation an. Im Sinne von Open Innovation können Privatkunden formiert als Community sowie externe Partner eigene Module auf Basis des Ausbausystems entwickeln. Diese können wiederrum über die Plattform für dem Endkunden zum Kauf bzw. zur Benutzung zur Verfügung gestellt werden. Dieses Vorgehen lässt sich mit dem Konzept von Open Source aus der Software-Entwicklung vergleichen (Perens, B.: 1999). 


\section{Literaturverzeichnis}

Bendapudi, N. \& Robert P. L. (2003):. Psychological implications of customer participation in co-production. In: Journal of marketing 67 (1), 14-28.

Perens, B. (1999): The open source definition. Open sources: voices from the open source revolution, 1, 171-188.

Scheibehenne, B., Greifeneder R. \& Todd P. M. (2010): Can there ever be too many options? A meta-analytic review of choice overload. In: Journal of consumer research 37 (3), 409-425.

Zukunftsinstitut (2020): Die Megatrend-Map. https://www.zukunftsinstitut.de/artikel/die-megatrend-map/, veröffentlicht 2020, abgerufen am 11.03.2021.

\section{Kontakt}

Dipl.-Ing. Linda Geißler

Dr. Nico Herzberg

Campeleon $\mathrm{GmbH}$

Gostritzer Straße 61

01217 Dresden

www.campeleon.com 